\title{
Magnetic Small World Nanomaterials: Physical Small World Networks
}

\author{
M.A. Novotny X. Zhang, J. Yancey, T. Dubreus, M.L. Cook, S.G. Gill, I.T. Norwood, and A.M. Novotny \\ Department of Physics and Astronomy, ERC Center for Computational Sciences, \\ Mississippi State University, Mississippi State, Mississippi 39762-5167 \\ G. Kornis: \\ Department of Physics, Applied Physics, and Astronomy, \\ Rensselaer Polytechnic Institute, Troy, NY 12180-3590
}

(Dated: July 9, 2018)

\begin{abstract}
The question addressed is whether magnetic materials based on physical small world networks are possible. Physical constraints, such as uniform bond length and embedding in three dimensions, are the new features added to make small world networks physical. Results are presented to further determine if physical small world networks can exist, and the effect of the small world connections on the critical phenomena of Ising models on such networks. Spectra of the Laplacian on randomlycollapsed bead-chain networks are studied. The scaling function for the order parameter of an Ising model with physical small world connections is presented.
\end{abstract}

\section{INTRODUCTION}

It is well known that novel magnetic behavior is present in systems governed by fixed points in other than three dimensions, such as quasi-one-dimensional and quasi-two-dimensional magnetic systems $\stackrel{\underline{1}}{\underline{1}}$ Recently there has been renewed interest in the behavior of complex networks, ${ }_{2}^{2}$ including small-world (SW) networks $\stackrel{3}{3}$ These networks mainly originate in areas in technology,

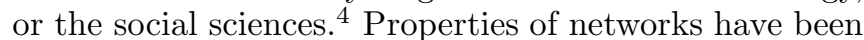
used in applications, for example, to devise perfectly scalable parallel algorithms ${ }^{5}$ Magnetic models, including Ising 6.7.8.9.10.11,12 models, have been studied on such networks. Recently, the question has been asked whether or not materials based on physical SW networks are possible $\stackrel{13}{=}$ Physical constraints, such as uniform bond lengths and node sizes, and that the network must be embedded in three dimensions, are the new features we add to SW networks to make them physical ${ }^{13}$ Since magnetic models on such SW networks are usually governed

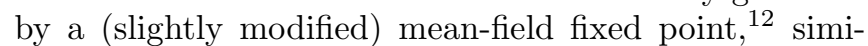
lar to mean-field fixed points for polymer systems magnetic spins on physical SW networks should exhibit novel magnetic behavior.

Here we present results to further determine whether physical SW networks can exist, and the effect of the SW connections on the critical phenomena of Ising models on such networks. We present results of a project with randomly-collapsed bead-chains to determine the topological properties and the properties of the Laplacian on such networks. The Laplacian operator is the most fundamental operator governing physical behavior on the network, such as diffusion or collective excitations in various interacting systems $\stackrel{17.18}{1}$ The starting lattice for the Ising simulations are either one-dimensional chains or two-dimensional toruses, with the physical SW connections added to the underlying lattice. We show, for example, how the mean-field behavior of Ising ferromagnets arises as the lattice size and density of SW connections is varied.

\section{MAGNETIC MODELS}

The magnetic models simulated have Ising spins, $S_{i}= \pm 1$, placed on the sites of a one $(d=1)$ or two dimensional $(d=2)$ square lattice, with periodic boundary conditions and nearest neighbor ferromagnetic exchange coupling $J_{1}$. A selected number of SW bonds, with ferromagnetic coupling $J_{2}=4 J_{1}$, are also included (Fig. 1). The Hamiltonian is

$$
\mathcal{H}=-J_{1} \sum_{\langle i, j\rangle} S_{i} S_{j}-J_{2} \sum_{\mathrm{sw}} S_{\mathrm{sw} i} S_{\mathrm{sw} j} .
$$

Our Monte Carlo simulations $\frac{19}{19}$ use a Glauber dynamic with random, single-site updates. When there are no SW bonds for the $d=2$ model the critical temperature $k_{B} T_{c} \approx$ $2.269 J_{1}$. A modified Wang-Landau method ${ }^{20}$ has been used to obtain comparisons with standard Monte Carlo results.

\section{PHYSICAL MODELS}

Two methods to construct physical networks, close to SW networks, were used. In both cases, the networks formed a physical network because the network is embedded in $d=3$ in such a way that all bond lengths are short-ranged and nearly equal.

In the first method, four high school students sequentially numbered all beads, randomly collapsed, and glued Mardi Gras necklaces of two different lengths. The bead size was $8 \mathrm{~mm}$, and the lengths of the necklaces were $106 \mathrm{~cm}$ (about 60 beads) and $254 \mathrm{~cm}$ (about 155 beads). Beads were cut from the necklaces, with a record kept of the adjacency matrix, $\mathbf{A}$. The matrix $\mathbf{A}$ has 1 as its $i, j$ element if bead $i$ is connected (either by the necklace string or by glue) to bead $j$. Otherwise the matrix element is zero.

Physical SW networks were also constructed by implementing a simulated annealing program to find an energy minimum of an arrangement of atoms starting with 

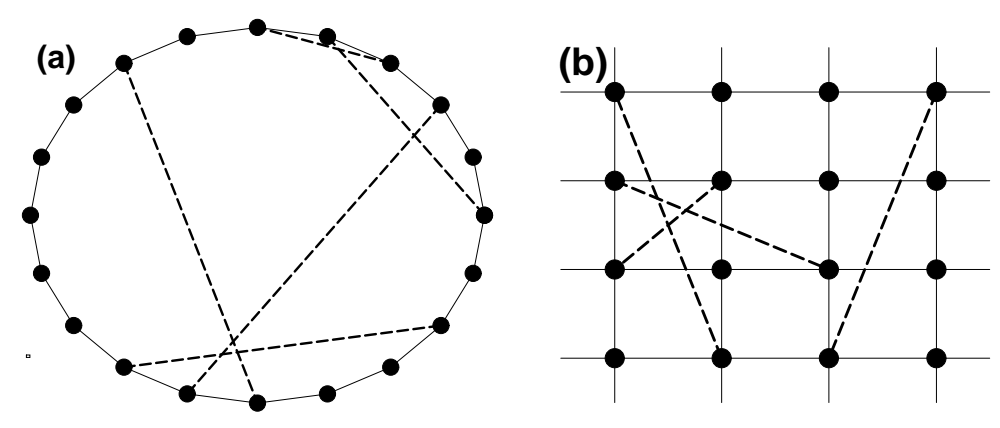

FIG. 1: Examples of the lattices with SW connections that were studied. The filled circles are the locations of the Ising spins, the light solid bonds have strength $J_{1}$ and the dashed bonds (the SW bonds) have strength $J_{2}$. (a) A one-dimensional SW graph with $N=20$ and five randomly chosen SW bonds. (b) A square-lattice with $L=4$ with $L$ SW bonds.

a lattice such as in Fig. 1(a). The energies included were those due to the bond lengths, bond angles, dihedral angles, a Lennard-Jones interaction, and partial ionization. Energy parameters were taken from the AMBER 21 data set. These physical SW networks will be analyzed elsewhere, but lead to the question of how the number of SW bonds scale with the system size.

\section{DATA AND ANALYSIS}

The ordered (non-zero) eigenvalues of the (negative) Laplacian on the glued Mardi Gras bead-chain networks, $-A_{i j}+\delta_{i j} \sum_{\ell} A_{i \ell}$ [Fig. 2], were found to be very similar to those of SW networks, such as in Fig. 1(a). In particular, the value of the smallest eigenvalues (governing, e.g., low-energy excitations of such systems) are significantly larger than those in the spectrum of a pure 1d necklace (where with $N$ sites the eigenvalues are $2[1-\cos (2 \pi k / N)]$ with $k=0,1,2, \cdots, N-1)$. This indicates the opening of a pseudo-gap ${ }^{17.18}$ and the corresponding mean-field-like behavior in the physics of fused 1d systems, commonly observed in SW networks $\underline{\underline{12}}$

For physical SW networks, one unanswered question is how the number of SW bonds scales with the system size. Consequently, we have investigated cases in which a finite lattice has a reasonable number of SW bonds, but the density of SW bonds goes to zero as the system size increases. The order parameter $m$ should scale $\frac{19}{19}$ as $m=L^{-\beta / \nu} F(y)$ with $y=L^{1 / \nu} t=L^{1 / \nu}\left|\left(T-T_{\mathrm{c}}\right) / T_{\mathrm{c}}\right|$. For large $y$ with $L \rightarrow \infty$ and $t \ll 1$ the scaling function should asymptotically be $F(y) \approx B y^{\beta}$ for $T<T_{\mathrm{c}}$ and $F(y) \approx B^{\prime} y^{\beta-\nu}$ for $T>T_{\mathrm{c}}$. Since the density of SW bonds goes to zero as $L \rightarrow \infty$, the critical exponents and critical temperature should be those of the underlying
Ising model. Fig. 3(a) shows the scaling function for $m$ for a $L \times L$ square lattice with no SW bonds, and Fig. 3(b) for $L \mathrm{SW}$ bonds of strength $J_{2}=4 J_{1}$. Although asymptotically the agreement with the normal Ising exponents is apparent, the SW bonds modify the scaling function. This illustrates that some properties of the mean-field behavior due to SW bonds $\frac{6.12 .13}{}$ should be observed in these finite (hundreds of nanometer in length) systems.

\section{CONCLUSION}

We have presented initial investigations of physical SW networks, and the magnetic behavior caused by SW interactions in Ising systems. Further work is required to definitively illustrate the behavior of magnetic systems on physical SW networks. Even if physical SW connections are shown to be irrelevant in the thermodynamic limit, the scaling functions for nanomaterials change substantially, providing quasi- SW behavior. Consequently, the current known results should provide a strong impetus to attempt to synthesize (or construct) and measure magnetic properties of such systems.

\section{ACKNOWLEDGEMENTS}

Useful conversations are acknowledged with P.A. Rikvold and Z. Toroczkai. This work was supported by NSF Grants No. DMR-0120310, DMR-0113049, and DMR-0426488, and by the ERC Center for Computational Sciences at Mississippi State University. The high school students (M.L.C., S.G.G, I.T.N., A.M.N) thank T. Breckenridge and the ERC for its hospitality.
* Electronic address: novotny@erc.msstate.edu

$\dagger$ Electronic address: korniss@rpi.edu

1 Low Dimensional Conductors and Superconductors,
NATO ASI Series B, Phys. Vol. 155, editor D. Jerome and L.G. Caron (Plenum, New York, 1987).

2 R. Albert and A.-L. Barabási, Rev. Mod. Phys. 74, 47 

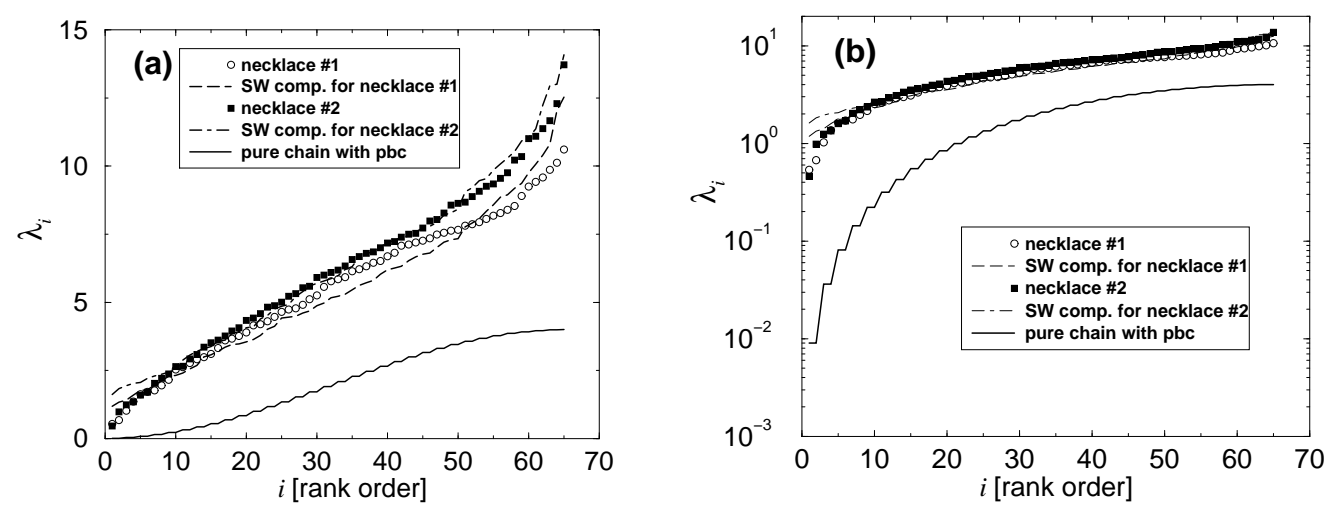

FIG. :

corres

lattice
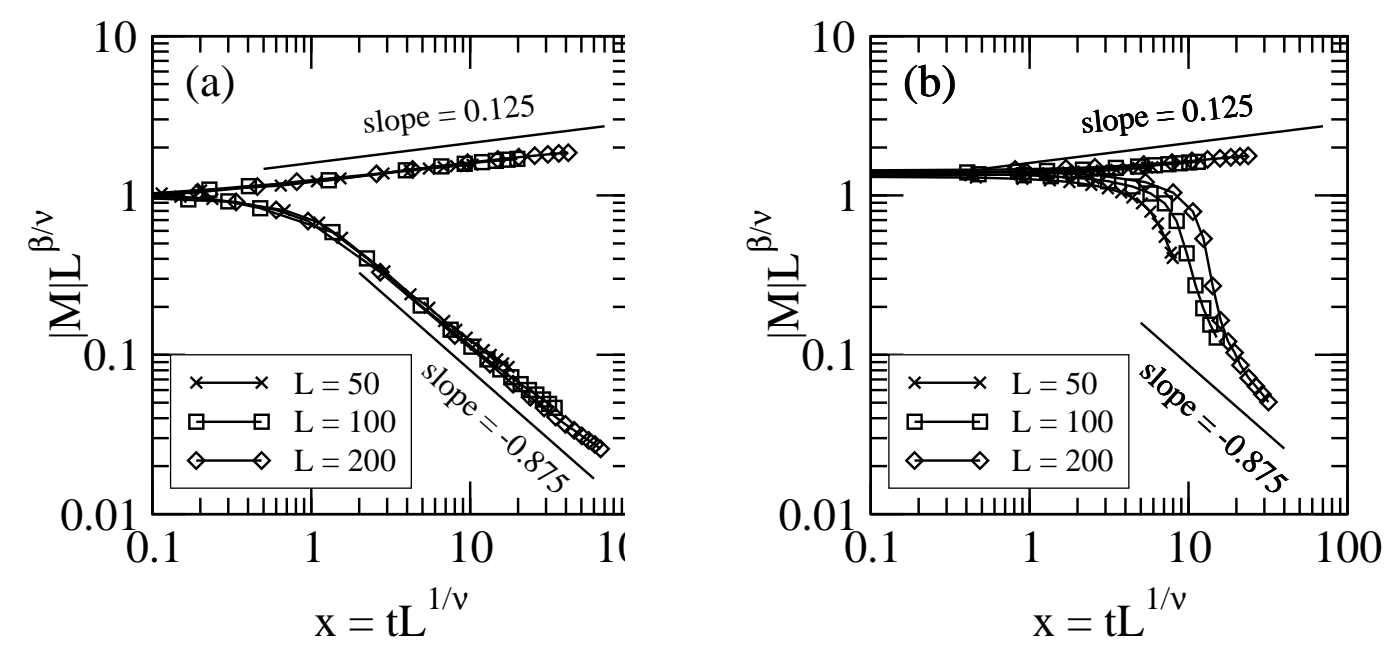

FIG. 3: The scaled order parameter for Ising models on $L^{2}$ square lattices. The asymptotic slopes have values of $\beta=\frac{1}{8}$ and $\beta-\nu=-\frac{7}{8}$. (a) for no SW connections, (b) for $L$ SW connections, as in Fig. 1(b), with strength $J_{2}=4 J_{1}$.

(2002).

3 D.J. Watts and S.H. Strogatz, Nature 393, 440, 1998.

4 M.E.J. Newman, SIAM Review 45, 167, 2003.

5 G. Korniss, M.A. Novotny, H. Guclu, Z. Toroczkai, and P.A. Rikvold, Science 299, 677 (2003).

${ }^{6}$ M. Gitterman, J. Phys. A 33, 8373 (2000).

7 A. Barrat and M. Weigt, Eur. Phys. J. B 13, 547 (2000).

8 B.J. Kim, H. Hong, P. Holme, G.S. Jeon, P. Minnhagen, and M.Y. Choi, Phys. Rev. E 64, 056135 (2001).

9 C.P. Herrero, Phys. Rev. E 65, 066110 (2002).

${ }^{10}$ H. Hong, B.J. Kim, and M.Y. Choi, Phys. Rev. E 66, 018101 (2002).

11 D. Jeong, H. Hong, B.J. Kim, and M.Y. Choi, Phys. Rev. E 68, 027101 (2003).

12 M.B. Hastings, Phys. Rev. Lett. 91, 098701 (2003).

13 M.A. Novotny and S.M. Wheeler, Brazilian J. Phys. 34,
395 (2004).

14 S. Janssen, D. Schwahn, and T. Springer, Phys. Rev. Lett. 68, 3180 (1992).

15 K.K. Mon and K. Binder, Phys. Rev. B 48, 2498 (1993).

16 E. Luijten and K. Binder, Phys. Rev. E 58, R4060 (1998).

17 R. Monasson, Eur. Phys. J. B 12, 555 (1999).

18 B. Kozma, M.B. Hastings, G. Korniss, Phys. Rev. Lett. 92, 108701 (2004).

19 D.P. Landau and K. Binder, A Guide to Monte Carlo Simulations in Statistical Physics (Cambridge University Press, Cambridge, UK, 2000).

${ }^{20}$ F. Wang and D.P. Landau, Phys. Rev. Lett. 64, 056101 (2001).

21 See http://amber.scripps.edu 